\title{
Predictors of recurrent TB in sputum smear and culture positive adults: a prospective cohort study
}

\author{
Grace Muzanyi. ${ }^{1}$, Y Mulumba $^{2}$, Paul Mubiri, ${ }^{1}{ }_{\text {Harriet Mayanja, },{ }^{1}, \text { John L Johnson }}{ }^{1,3}$, Ezekiel Mupere ${ }^{1}$
}

1. Uganda-Case Western Reserve University Research Collaboration, Kampala, Uganda.

2. Uganda Cancer Institute, Kampala, Uganda.

3. Tuberculosis Research Unit, Department of Medicine, Case Western Reserve University and University

Hospitals Case Medical Center, Cleveland, Ohio, U.S.A.

4. Makerere University, School of Public Health.

\begin{abstract}
Objective: To explore simple inexpensive non-culture based predictors of recurrent pulmonary tuberculosis (PTB).

Setting and study population: HIV-infected and uninfected adults with the first episode of smear positive, culture-confirmed pulmonary tuberculosis in a high tuberculosis burden country.

Design: A nested prospective cohort study of participants with pulmonary tuberculosis (PTB) presenting to a hospital out-patient clinic.

Results: A total of 630 TB culture confirmed participants were followed up for eighteen months of which $57(9 \%)$ developed recurrent recurrent TB. On univariate analysis, $4.7 \%$ low grade $(1+)$ pre-treatment sputum smear participants developed recurrent tuberculosis Vs $8.8 \%$ with high grade $(3+)$ smears $(\mathrm{OR}=0.31,95 \% \mathrm{CI}: 0.10-0.93, \mathrm{p}=0.037)$. On multivariate analysis:participants with extensive fibro-cavitation had a high risk of recurrent TB Vs minimal end of treatment fibro-cavitation $(18 \% \mathrm{Vs} 12 \%, \mathrm{OR}=2.3,95 \% \mathrm{CI}: 1.09-4.68, \mathrm{p}=0.03)$. Weight gain with HIV infection was assosciated with a high risk of recurrent TB Vs weight gain with no HIV infection $(18 \% \mathrm{Vs} 6 \%, \mathrm{OR}=6.8,95 \% \mathrm{CI}: 165-27.83, \mathrm{p}=0.008)$ where as weight gain with a low pre-treatment high bacillary burden was assosciated with a low risk of recurrent TB Vs weight gain with a high pre-treatmentbacillary burden $(6.5 \% \mathrm{Vs} 7.9 \%, \mathrm{OR}=0.2,95 \% \mathrm{CI}: 0.05-0.79, \mathrm{p}=0.02)$.

Conclusion: Extensive end of treatment pulmonary fibro-cavitation, high pre-treatment bacillary burden with no weight gain and HIV infection could be reliable predictors of recurrent tuberculosis.

Keywords: Grade, fibrosis, cavities, weight.

DOI: https://dx.doi.org/10.4314/ahs.v19i2.33

Cite as: Muzanyi G, Mulumba Y, Mubiri P, Mayanja H, Johnson JL, Mupere E. Predictors of recurrent TB in sputum smear and culture positive adults: a prospective cohort study. Afri Health Sci.2019;19(2): 2091-2099. bttps:// dx.doi.org/10.4314/abs.v19i2.33
\end{abstract}

\section{Introduction}

Long term, non-relapsing cure is the ultimate goal of anti-TB treatment; unfortunately recurrent disease(relapse/ re-infection) occurs on average in about two to six percent of patients with drug-susceptible TB treated with modern rifampicin-containing short course regimens ${ }^{12}$. Recurrent TB may result from recurrent disease with the

\section{Corresponding author: \\ Grace Muzanyi, Uganda-Case Western Reserve University \\ Research Collaboration, P.O. Box 663, Kampala, Uganda. \\ Phone: +256772 437028 . \\ Email: gxm62@case.edu}

original or new infection strain of $M$. tuberculosis ${ }^{28}$.This may be due to several scenarios that include: poor adherence, immunosuppression mainly due to HIV co-infection $^{10,13,28}$, Initial TB infection with a high bacillary burden ${ }^{17}$, drug resistant strains ${ }^{30}$, sub-optimal exposure in fixed dose combinations ${ }^{1}$, variation in biological response to $\mathrm{TB}$ treatment $^{31}$ or exogenous re-infection with a new strain $^{23}$.

Clinicians have sought to identify factors associated with an increased risk for recurrent TB after successful initial treatment. This can guide them in identifying patients and groups where treatment might be modified or where post-treatment follow-up might be useful. Lack of weight gain $^{8}$, HIV infection ${ }^{13}$, pre-treatment greater radiographic involvement ${ }^{9}$, pre-treatment high bacillary burden ${ }^{17}$,post treatment residual fibro-cavitation ${ }^{9}$, sub-optimal exposure

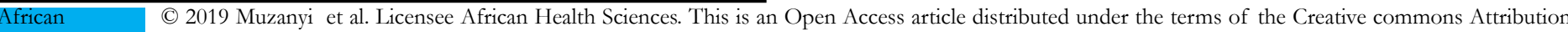

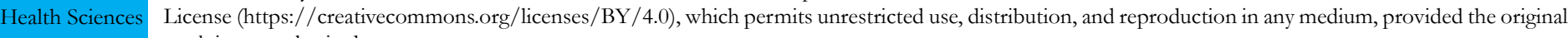
work is properly cited.
} 
in fixed dose combinations ${ }^{1}$, cavitary disease on chest radiograph\& a positive sputum culture after completing the first two months of anti-TB treatment have been associated with recurrent tuberculosis.

Sputum culture is costly and less affordable in many high TB burden settings ${ }^{26}$. Genexpert is as well costly and less specific in diagnosis of recurrent $\mathrm{TB}^{26,27}$. There is a programmatic intuitive need to identify simple, robust, inexpensive, convenient, non culture (non-molecular) based tools that rely on readily available assessments at the point of care to identify patients at risk for recurrent TB. Most TB suspects have a chest radiograph ${ }^{31}$ and sputum smear done during evaluation ${ }^{33}$. Patients with newly diagnosed TB are offered HIV testing by most programmes ${ }^{32}$ and even peripheral TB units have a weighing balance to measure body weight for anti-TB drug dosing. Sputum smear microscopy is a commonly used TB diagnostic tool even in resource constrained settings ${ }^{33}$. To our knowledge, no previous study has ever come up with a predictive non-culture based tool for recurrent TB from the list of previously identified risk factors. In this prospective cohort study, we examined whether one or any combination of weight gain, sputum smear grade, pre-treatment cavitation, end of treatment residual fibro-cavitation, radiographic extent of TB disease and HIV co-infection could form a non-culture based a predictive tool to identify patients at risk for recurrent TB in the initial eighteen months post-treatment in a well-followed up treatment cohort in Uganda, a nation with a high burden of TB.

\section{Methods}

\section{Study population and setting}

The participants included in this prospective cohort study were systematically selected from two parent studies. The parent studies recruited participants from several health units within a radius of 30 kilometers from the research Site in Kampala. This analysis included data from HIV-infected and -uninfected adults over 18 years-old with the first episode of smear positive, culture-confirmed, drug-susceptible pulmonary TB, treated in the Kawempe study(DMID protocol number:01-005) conducted from April 2002 to July2012 and the EDCTP TB diagnostics study(EDCTP - IP_09_32040_011)conducted from March 2010 through March 2013.Both studies were conducted at a large out-patient TB clinic within
Mulago Hospital, a national tertiary referral hospital in Kampala,Uganda . All patients were treated with self-administered, standard short course therapy (2 months of daily INH, rifampicin, ethambutol and pyrazinamide followed by daily treatment with Isoniazid and rifampicin for 4 months). The TB drugs were dispensed as single drugs. ${ }^{1}$ The Kawempe study enrolled TB index cases and followed up their household contacts. The Kawempe study embedded a mixture of cohort and cross sectional designs. A Kawempe community resident was defined as one who should have resided in the Kawempe area for at least three months. The Kawempe study excluded participants with recurrent TB and those who had taken more than 5 doses of anti-TB drugs for that episode of TB at the time of enrolment.Participants were followed up for eighteen months post TB treatment. A team of home health visitors ensured routine weekly field visits, phone calls and continued counseling of the participants to mitigate loss to follow up. The EDCTP TB diagnostics study was a prospective cohort study that evaluated TB cytokine signatures in blood, sputum and urine as markers of active TB infection in relation to sputum culture. It enrolled both controls and active TB cases, first episode of $\mathrm{TB}$ and excluded those who had taken more than 5 doses of anti-TB drugs.. All culture confirmed TB cases from both parent studies were systematically selected and screened for this analysis. Data was extracted using a tested and validated questionnaire by two staffs who were blinded to the study objective.

\section{Procedures}

HIV infection was defined as having a positive result on three approved, licensed rapid HIV testing kits in a validated HIV testing algorithm.Each participant had a posterior-anterior chest radiograph taken pre-treatment, end of treatment and at the time of suspected TB recurrence. The radiographic severity of $\mathrm{TB}$ disease was assessed as minimal, moderate or far advanced disease using the scheme of the US National TB and Respiratory Disease Association $^{16}$. Body weight was measured at baseline and at each follow up visit using a calibrated beam balance (Detecto model). Sputum smear and culture were done monthly during the treatment phase, every 6 months during follow up and at the time of suspected recurrent TB. Sputum smears were done using fluorescent auramine staining. Smear grading was by the World Health Organi- 
zation scale ${ }^{20}$. Sputum culture was on Lowenstein-Jensen slants. Mycobacterium tuberculosis speciation was by polymerase chain reaction $(\mathrm{PCR})^{24}$. Initial isolates had drug susceptibility testing done for INH, rifampicin, ethambutol, pyrazinamide and streptomycin.

All participants with suspected recurrent TB were evaluated by clinical examination, chest radiography, multiple sputum smears and cultures. Recurrent TB was defined as culture confirmed TB during 18 months of follow up in participants who were microbiologically confirmed cured at the end of the initial six months of TB treatment. Weight gain was defined as gaining at least $5 \%$ of the pre-treatment body weight by the end of the first two months of anti-TB treatment ${ }^{8}$.

Treatment adherence was monitored by urine INH metabolite testing in addition to participant interview at each scheduled visit and review of attendance records. Defaulters were traced by home health visitors. All participants were followed up for 18 months after TB treatment. Both parent studies were approved by institutional review boards of the Joint Clinical Research Centre, the National AIDS Research Committee and the Ugandan National Council for Science and Technology. All participants gave written informed consent for participation in the parent studies.

\section{Statistical analysis}

Categorical data was analyzed using the chi-square. For weight gain, changes from baseline by month 2 were as- sessed using paired z-tests. Logistic regression was used in determining the association between weight gain, HIV status, radiographic extent of disease, sputum smear grade, end of treatment fibro-cavitation and the likelihood of TB recurrence.Stepwise selection method of predictor variables(weight gain,HIV status, chest x-ray extent of disease,fibro-cavitation and bacillary burden) was used for inclusion in the logistic model. All tests were two-sided. The odds ratio plus the corresponding 95\% confidence interval and $\mathrm{p}$-value were the effect measure. A confidence interval excluding the null value of 1 and a $\mathrm{p}$-value $<0.05$ were considered statistically significant. All statistical analyses were done using Stata (Version 12, STATA, USA).

\section{Results}

A total of 1150 ( figure 1 ) were screened and 690 bacteriologically confirmed PTB study participants were enrolled in the two parent studies between April 2001 and July 2012 of which 60 were excluded from this analysis since they were not followed up after completing treatment. Of the 630 followed up, 57 (9\%) developed recurrent TB, 136(22\%) were HIV infected, 311(49\%) had far advanced disease on chest radiograph \& 363(58\%) had pulmonary cavitation. Majority $331(52 \%)$ were female $\&$ recurrent TB cases were older (mean age $=30 \mathrm{yrs}$, $\mathrm{SD}=10.8$ :) Vs the non- recurrent (NR) group (mean age 28.6yrs: $\mathrm{SD}=10.2) .106(17 \%)$ had a low grade pretreatment smear \& $160(25 \%)$ had a high grade smear. Majority $510(81 \%)$ had urine INH testing to confirm TB treatment adherence. 


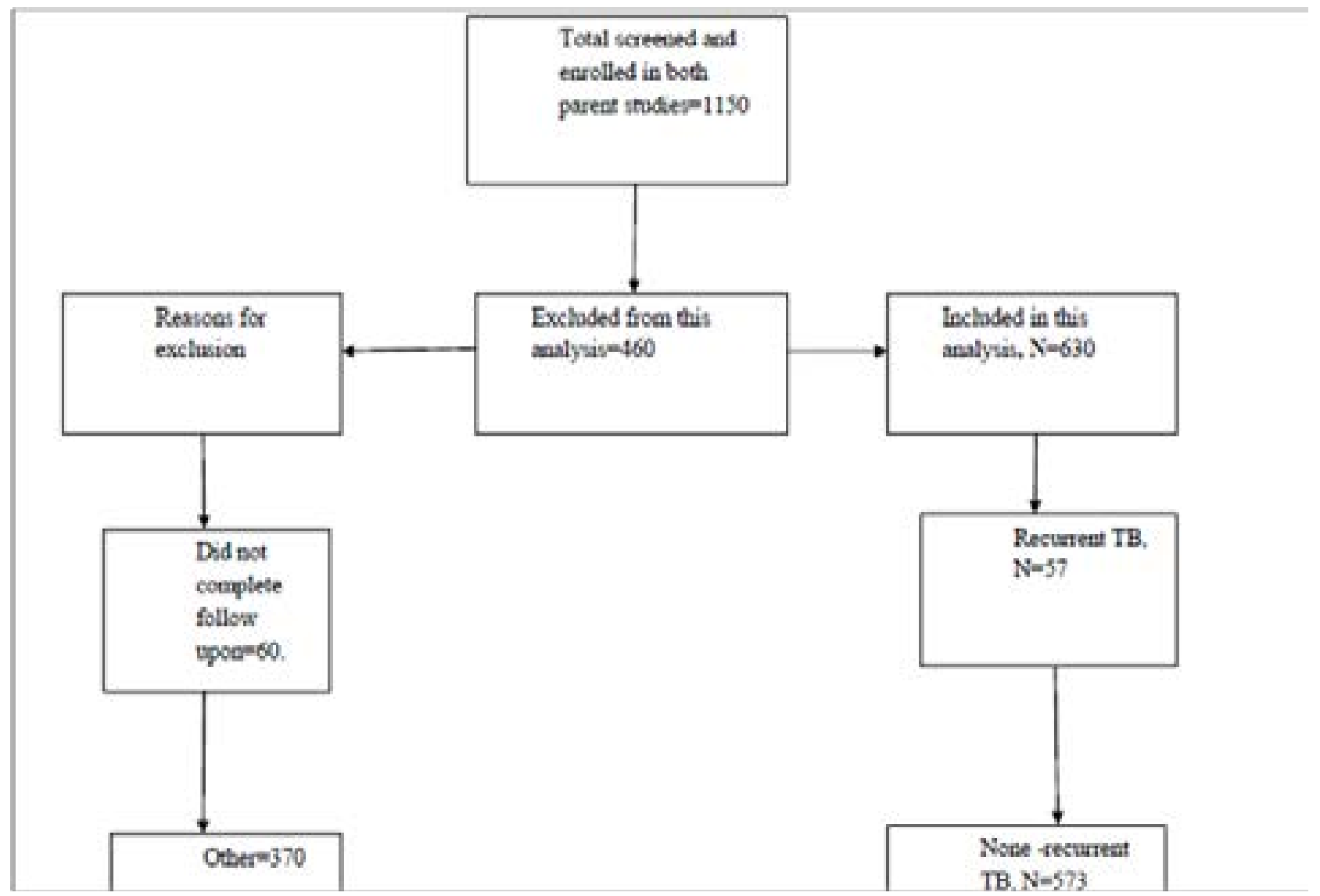

Figure 1: Study profile 
Table 1: Univariate analysis

\begin{tabular}{|c|c|c|c|c|}
\hline Variable & Recurrent & Non-Recurrent & Total & P-value \\
\hline $\begin{array}{l}\text { Weight gain } \\
<5 \%\end{array}$ & 38 & 335 & 362 & 0.45 \\
\hline Females & 28 & 303 & 331 & 0.64 \\
\hline Alcohol & 04 & 79 & 083 & 0.17 \\
\hline Smoking & 09 & 81 & 090 & $\overline{0.71}$ \\
\hline Age:mean(SD) & $30(11)$ & $29(10)$ & --- & 0.22 \\
\hline HIV positive & 12 & 124 & 136 & 0.92 \\
\hline $\begin{array}{l}\text { Baseline cavities } \\
\text { Chest x-ray } \\
\text { extent of disease } \\
\text { Minimal }\end{array}$ & 38 & 325 & 363 & 0.15 \\
\hline Moderate & 5 & 58 & 63 & 0.48 \\
\hline Advanced & $\begin{array}{l}16 \\
34\end{array}$ & $\begin{array}{l}193 \\
277\end{array}$ & $\begin{array}{l}209 \\
311\end{array}$ & $\begin{array}{l}0.45 \\
0.15\end{array}$ \\
\hline Smear & & & & \\
\hline $1+$ & 05 & 101 & 106 & 0.032 \\
\hline $2+$ & 16 & 109 & 125 & 0.844 \\
\hline $3+$ & 14 & 146 & 160 & -----' \\
\hline
\end{tabular}

On univariate analysis(table 1),4.7\% of the low grade $\mathrm{p}=0.037$ ). Weight gain and HIV infection did not indesmear participants developed recurrent TB Vs 8.8\% pendently influence the rate of recurrent TB on univariwith a high grade smear (OR=0.31, 95\%CI: 0.10-0.93, ate analysis. On multivariate analysis:table 2;

Table 2: Multivariate analysis

\begin{tabular}{lllll}
\hline Variable & Recurrent & Non-Recurrent & Total 95\%CI & P-value \\
\hline Weight gain $<5 \%$,HIV+ & 5 & 80 & $850.18-1.3$ & 0.14 \\
$\begin{array}{l}\text { Weight gain } \geq 5 \% \text {,HIV- } \\
\begin{array}{l}\text { Weightgain } \geq 5 \% \text {,moderate } \\
\text { disease }\end{array}\end{array}$ & 11 & 165 & $1761.7-27.8$ & 0.008 \\
$\begin{array}{l}\text { Weightgain } \geq 5 \% \text {,advanced } \\
\text { disease }\end{array}$ & 12 & 44 & $490.14-20.66$ & 0.68 \\
$\begin{array}{l}\text { HIV-,moderate disease } \\
\text { HIV-,advanced disease }\end{array}$ & 15 & 122 & $1340.15-16.84$ & 0.69 \\
Smear 1+, Weight gain $\geq 5 \%$ & 39 & 159 & $1740.6-6.8$ & 0.23 \\
& 3 & 268 & $3070.46-26.9$ & 0.20 \\
& 70 & $730.05-0.79$ & 0.02
\end{tabular}


weight gain with a low grade smear was associated with an even lower risk of recurrent TB Vs weight gain with a high pre-treatment bacillary burden $(\mathrm{OR}=0.2,95 \% \mathrm{CI}$ : 0.05-0.79,P=0.02):. Weight gain with HIV infection was associated with a high risk of recurrent TB Vs weight gain with no HIV infection $(18 \% \mathrm{Vs} 6 \%$, OR=6.8,95\%CI: 165 $27.83, \mathrm{p}=0.008$. About $18 \%$ of those with extensive fibro-cavitary sequellae at the end of TB treatment developed recurrent TB Vs 12\% with minimal fibro-cavitary sequellae $(\mathrm{OR}=2.3,95 \% \mathrm{CI}$ : 1.09-4.68, $\mathrm{P}=0.03)$ : table 3 .

Table 3: Radiographic extent of disease at month 6(fibro-cavitation)

\begin{tabular}{|c|c|c|c|c|}
\hline Variable & Recurrent & Non-Recurrent & Total $95 \% \mathrm{CI}$ & P-value \\
\hline Minimal & 16 & 123 & $\begin{array}{l}139 \\
\end{array}$ & ---- \\
\hline Moderate & 15 & 108 & $123 \quad 0.7-2.8$ & 0.33 \\
\hline Advanced & 13 & 58 & $\begin{array}{ll}71 & 1.1-4.7\end{array}$ & 0.03 \\
\hline
\end{tabular}

\section{Discussion}

In this analysis,we report a 2 fold reduction in the risk of recurrent TB among participants with low grade compared to high grade pre-treatment smears $(4.7 \% \mathrm{Vs} 8.8 \%)$ and inversely an increase in the risk of recurrent TB among participants with extensive end of treatment fibro-cavitary sequellae compared to those with minimal fibro-cavitary sequellae $(18 \% \mathrm{Vs} 12 \%)$. Rupka Singla et al 2007 and panJab et al 2007 showed that high pre-treatment bacillary burden and residual cavitation by the end of TB treatment increases the risk for recurrent TB. Luzze et al 2013 showed that extensive end of treatment fibrosis and cavitation was associated with recurrent TB. Therefore our findings are similar to what has been reported in previous studies ${ }^{9,17,28}$. Participants with a low grade pretreatment smear and weight gain had an even lower risk of recurrent TB but weight gain alone did not independently influence the risk of recurrence 8 . We however observed a high proportion of failed weight gain in the recurrent TB group compared to their non-recurrent counter parts (67\% Vs 59\%) but this was not statistically significant although Khan et al $2006^{8}$ showed that failure to gain $\geq 5 \%$ of baseline body weight by the end of the intensive phase of TB treatment was associated with an increased risk for TB recurrence. Our study found no statistically significant difference on univariate analysis although there was a higher proportion of failed weight gain in the recurrent Vs the non-recurrent group. We also observed an increased risk of recurrent TB among the HIV infected with weight gain Vs HIV uninfected with weight gain. Previous studies have shown increased rates of TB recurrence among HIV-TB patients ${ }^{10}$, this was the case with our study results too. We noted a higher pro- portion of recurrent TB (9\%) in this population contrary to previously existing scientific data ${ }^{12}$ however,our results are in line with the findings of one previous study in the same population ${ }^{28}$. The high proportion of recurrent TB could be attributed to patients presenting late with advanced PTB $^{9}$ as evidenced in this analysis where the majority $(49 \%)$ of the participants had radiographically far advanced disease. This is in agreement with Panjab et al 2007 findings of greater radiographic involvement being a risk factor for recurrent TB Urine INH metabolite testing was not done in 19\% of the cases in which case non-adherence could have confounded recurrent PTB ${ }^{29}$. We also noted that about $54 \%$ of the cases presented with a high pre-treatment bacillary burden which could partially explain the high proportions of recurrent $\mathrm{TB}$ in this population ${ }^{17}$.

The strength of our study is that it involved an eighteen month follow-up of a large cohort of confirmed TB patients and monthly assessment of adherence by urinary INH metabolite testing during treatment. Our study had several limitations (i) the urine INH metabolite testing was only possible in $81 \%$ of the cases included in this analysis. (ii)DNA finger printing was not done to distinguish recurrent $\mathrm{TB}$ due to relapse from exogenous reinfection with a different $\operatorname{strain}^{28}$.

\section{Recommendation}

Previous studies have listed several factors assosciated with TB disease recurrence but no predictive tool has been assembled out of this literature to influence management of recurrent TB. We recommend that clinicians should put into perspective the possibility of prolonging treatment and close patient monitoring for TB pa- 
tientswho :(i) present with a high pre-treatment bacillary burden $(3+)$ and failure to gain at least $5 \%$ of baseline body weight by the end of the intensive phase of TB treatment. (ii)Are HIV positive.(iii) have extensive end of treatment fibrosis and residual cavitation on chest $\mathrm{x}$-ray (grade 3).

\section{Acknowledgement}

The authors thank the study participants, Uganda-Case research collaboration project clinic staff and field workers, the Uganda National TB and Leprosy Programme and Mulago Hospital for their assistance in completing these long term studies.

\section{Funding}

The parent studies were funded by the Tuberculosis Research Unit at Case Western Reserve University, established with funds from the United States National Institutes of Allergy and Infectious Diseases, National Institutes of Health and Human Services, under contract NO1-AI95383 and HHSN266 200700022C/NO1AI-70022 and the European Developing Countries Clinical Trials Partnership (EDCTP) Grant number: EDCTP - IP_09_32040_011.

\section{Conflict of interest}

We have no conflict of interest to declare.

\section{References}

1. Pramudita A, Rumende CM, Findyartini A:Fixed-dose combination anti-tuberculosis therapy as a risk factor for tuberculosis recurrence: an evidence based case report: ActaMedIndones. 2017Apr;49(2):175-182:PMID:28790234 2. Kopanoff, D E Snider, Jr, and M Johnson: Recurrent tuberculosis: why do patients develop disease again? United States Public Health Service cooperative survey Division of Tuberculosis Control, Centers for Disease Control, Atlanta, GA 30333:

3. Pettit AC1, Kaltenbach LA, Maruri F, Cummins J, Smith TR, Warkentin JV, Griffin MR, TR: Chronic lung disease and HIV infection are risk factors for recurrent tuberculosis in a low-incidence setting. Int JTuberc Lung Dis. 2011 Jul; 15(7):906-

4. Sterling TR, Alwood K, Gachuhi R, et al. Relapse rates after short-course (6-Month) treatment of tuberculosis in HIV-infected and uninfected persons. AIDS. 1999; 13:1899-1904
5. Golub JE, Durovni B, King BS, et al. Recurrent tuberculosis in HIV-infected patients in Riode Janeiro, Brazil. AIDS. 2008; 22:2527-2533.

6. Cox H, Kebede Y, Allamuratova S, Ismailov G, Davletmuratova Z, et al. (2006) Tuberculosis recurrence and mortality after successful treatment: Impact of drug resistance. PLoS Med 3(10): e384. DOI: 10.1371/journal. pmed.0030384.

7. Nyamogoba H DN1, 2, Kikuvi G2, Mbuthia G3, Mpoke S4, Obala A A1, Biegon R1etal: A high rate of recurrent tuberculosis in western Kenya independent of human [I Immunodeficiency virus infection: Afr J Health Sci. 2012; 20:62-68

8. Awal Khan, Timothy R. Sterling, Randall Reves, Andrew Vernon, C. Robert Horsburgh, and the Tuberculosis Trials Consortium: Lack of Weight Gain and Relapse Risk in a Large Am J Respir Crit Care Med Vol 174. pp 344-348, 2006. Tuberculosis Treatment Trial: Am J

9. Panjabi R1, Comstock GW, Golub JE: Recurrent tuberculosis and its risk factors: adequately treated patients are still at high risk: Int J Tuberc Lung Dis. 2007 Aug;11(8):82837.

10. M Hawken, FRACP, a, P Nunn, MRCPa, P Godfrey-Faussett, MRCPa, K.P.W.J McAdam, FRCPa (Prof), $\mathrm{J}$ Morris, MScb, J Odhiambo etal:Recurrence of tuberculosis in HIV-1-infected patients in Kenya: The Lancet: Volume 342, Issue 8867, 7 August 1993, Pages 332-337: Originally published as Volume 2, Issue 8867

11. Falk A, O'Connor JB, Pratt PC, Webb WR, Wier JA, Wolinsky E. Chapter 6. Classification of pulmonary tuberculosis. In: Diagnostic standards and classification of $12^{\text {th }}$ Ed. New York: National Tuberculosis and Respiratory Disease Association; 1969. p. 68-76 tuberculosis.

12. Stephen E. Weis, Philip C. Slocum, Francis X. Blais, Barbara King, Mary Nunn, G. Burgis Matney: The Effect of Directly Observed Therapy on the Rates of Drug Resistance and Relapse in Tuberculosis:N Engl J Med 1994; 330:1179-1184April 28, 1994DOI: 10.1056/ NEJM199404283301702: 13. Int J TubercLung Dis. 2011 Jul; 15(7):906-11

13. Zhdanov V, Bilenko N, Mor Z. Risk Factors for Recurrent Tuberculosis among Successfully treated Patients in Israel, 1999-2011: Isr Med Assoc J. 2017 Apr;19(4):237241

14. Juan-Pablo Millet,1,2,3,* Evelyn Shaw,1 Àngels Orcau,1,2 Martí Casals,1,2,4,5 Jose M. Miró,6 Joan A. Caylà,1,2 and The Barcelona Tuberculosis Recurrence 
Working Groupł:Tuberculosis Recurrence after Completion Treatment in a European City: Reinfection or Relapse?: PLoS One. 2013; 8(6): e64898.

15. Blumberg HM, Burman WJ, Chaisson RE, Daley CL, Etkind SC, Friedman LN, Fujiwara P, Grzemska M, Hopewell PC, Iseman MD, Jasmer RM, Koppaka V,Menzies RI, O'Brien RJ, Reves RR, Reichman LB, Simone PM, Starke JR, Vernon AA; American Thoracic Society, Centers for Disease Control and Prevention and the Infectious Diseases Society: treatment of tuberculosis: $\mathrm{Am}$ J Respir Crit CareMed. 2003 Feb 15;167(4):603-62.

16. Falk A, O'Connor JB, Pratt PC, Webb WR, Wier JA, Wolinsky E. Chapter 6.

17. Rupak Singla, Neeta Singla,Rohit Sarin, Arora, V. K. (2005): Influence of Pre-Treatment Bacillary Load on Treatment Outcome of Pulmonary Tuberculosis Patients Receiving DOTS under RNTCP: Influence of Pre-Indian J Chest Dis Allied Sci, 47. pp. 19-23.

18. Diagnostic Standards and Classification of Tuberculosis. 12th Ed. New York: National Tuberculosis and Respiratory Disease Association; 1969. p. 68-76)..

19. Uganda national TB and leprosy control guidelines, $2^{\text {nd }}$ edition 2010

20. World Health organization (WHO) guidelines on the grading of sputum smear microscopy 2010 .

21. World health organization TB treatment guidelines 2010.

22. Pekovic V, Mayanja H, Vjecha M, Johnson J, Okwera A, Nsubuga P, Mugerwa R, Ellner J, Whalen C. Comparison of three composite compliance indices in a trial of self-administered preventive therapy for tuberculosis in HIV-infected Ugandan adults.. J Clin Epidemiol. 1998; 51:597-607)

23. Schiroli C1, Carugati M2, Zanini F2, Bandera A3, Di Nardo Stuppino S2, Monge E2, Morosi M2, Gori A3, Matteelli A4, Codecasa L5, Franzetti F2 :Exogenous reinfection of tuberculosis in a low-burden area: Infection. 2015 Dec; 43(6):647-53. Doi: 10.1007/s15010-015-07599. Epub 2015 Mar 10.

24. Maruthai K, Ravibalan T, Vallayyachari K, Kesavan S, Samrot AV, Muthaiah M.:Molecular typing and differentiation of Mycobacterium tuberculosis clinical isolates using Double Repetitive Element PCR and Duplex PCR:Int
J Mycobacteriol. 2015 Mar; 4(1):60-6. doi: 10.1016/j.ijmyco.2014.11.061. Epub2015 Jan 13:PMID: 26655200. Racil H, Ben Amar J, Mami M, Chabbou A:

25. Predictive factors for recurrence of pulmonary tuberculosis in Tunisia: a retrospective study. Rev Mal Respir. 2012 Mar; 29(3):412-8. doi: 10.1016/j.rmr.2012.01.001. Epub 2012 Feb 15. French: PMID: 22440306.

26. Rupert S, Vassall A, Raizada N, Khaparde SD, Boehme C, Salhotra VS, Sachdeva S, Nair SA, Hoog AH: Bottom-up or top-down: unit cost estimation of tuberculosis diagnostic tests in India: Int J Tuberc Lung Dis. 2017 Apr 1;21(4):375-380.doi:10.5588/ijtld.16.0496. PMID:28284251

27. Assessment of the sensitivity and specificity of Xpert MTB/RIF assay as an early sputum biomarker of response to tuberculosis treatment.Friedrich SO, Rachow A, Saathoff E, Singh K, Mangu CD, Dawson R, Phillips PP, Venter A, Bateson A, Boehme CC, Heinrich N, Hunt RD, Boeree MJ, Zumla A, McHugh TD, Gillespie SH, Diacon AH, Hoelscher M; Pan African Consortium for the Evaluation of Anti-tuberculosisAntibiotics(PanACEA): Lancet Respir Med. 2013Aug;1(6):46270.doi:10.1016/ S22132600(13)7011:4

28. Luzze H1, Johnson DF, Dickman K, Mayanja-Kizza H, Okwera A, Eisenach K, Cave MD, Whalen CC, Johnson JL, Boom WH, Joloba M; Tuberculosis Research Unit: Relapse more common than reinfection in recurrent tuberculosis 1-2 years post treatment in urban Uganda: Int J Tuberc Lung Dis. 2013 Mar;17(3):361-7. doi: 10.5588/ijtld.11.0692.

29. Cadosch D1, Abel Zur Wiesch P2,3, Kouyos R4, Bonhoeffer S1:The Role of Adherence and Retreatment in De Novo Emergence of MDR-TB: PLoS Comput Biol. 2016 Mar 11;12(3):e1004749. doi: 10.1371/journal. pcbi.1004749. eCollection 2016 Mar.

30. Goswami A1, Chakraborty U1, Mahapatra T2, Mahapatra S2, Mukherjee T3, Das S4, Das A2, Dey SK5, Ray S6, Bhattacharya B1, Pal NK: Correlates of treatment outcomes and drug resistance among pulmonary tuberculosis patients attending tertiary care hospitals of Kolkata, India: PLoS One. 2014 Oct 7; 9(10):e109563. doi: 10.1371/journal.pone.0109563. eCollection 2014.

31. Clifford V1, He Y1, Zufferey C1, Connell T1, Curtis 
$\mathrm{N} 2$ : Interferon gamma release assays for monitoring the response to treatment for tuberculosis: A systematic review: Tuberculosis (Edinb). 2015 Dec; 95(6):639-650. doi: 10.1016/j.tube.2015.07.002. Epub 2015 Jul 30

31. Aliyu G1, El-Kamary SS, Abimiku A, Hungerford L, Obasanya J, Blattner W:Cost-effectiveness of point-ofcare digital chest-x-ray in HIV patients with pulmonary mycobacterial infections in Nigeria. BMC Infect Dis. 2014 Dec 13;14:675. doi: 10.1186/s12879-014-0675-0.:
32. Thomas BE1, Ramachandran R, Anitha S, Swaminathan S:Feasibility of routine HIV testing among TB patients through a voluntary counseling and testing centre: Int J TubercLung Dis. 2007 Dec;11(12):1296-301

34. Myint O1, Saw S2, Isaakidis P3, Khogali M3, Reid A3, Hoa NB4,5, Kyaw TT6, Zaw KK2, Khaing TMM7, Aung ST7: Active case-finding for tuberculosis by mobile teams in Myanmar: yield and treatment outcomes: Infect Dis Poverty. 2017 Jun 2; 6(1):77. doi: 10.1186/s40249-017-0291-5. 